\section{Retrieval of information} \section{from organized memory sets*}

\author{
DONALD V. DeROSA and MARKETTA BECKWITH
}

Bowling Green State University, Bowling Green, Ohio 43403

A recognition reaction time (RT) investigation examined retrieval of individual elements from memorized sets of digits. The sets varied in organization and the delay between presentation of the items, and a recognition test was systematically manipulated. Organized sets produced faster reactions then unorganized sets. Serial position effects were less pronounced and operative for a shorter period of time with organized material.

Two recognition reaction time (RT) studies using four consecutive digits (e.g., $3,4,5,6$ ) as memory sets have yielded disparate results. DeRosa \& Morin (1970) used to-be-remembered sets of four conseculive digits (positive set) and observed that RTs to individual items varied as a function of the element's position in the set. They found that reactions were slowest to items that defined the boundaries of the positive set (e.g., 3 and 6 in the set $3,4,5,6)$, while interior items $(4,5)$ were responded to fastest. Their procedure involved identifying the positive set for $S$ prior to a long series of trials in which only test stimuli were shown. If the test item was a member of the positive set, $\mathbf{S}$ was instructed to say "yes," and "no" otherwise.

Morin, DeRosa, \& Stultz (1967) presented a new positive set serially on each trial, followed in $250 \mathrm{msec}$ by a test item. In one condition the positive sets were always four consecutive digits presented in ascending order. When RTs in this condition were plotted as a function of the position of the item in the positive set, the function was markedly different from the curves observed by DeRosa \& Morin (1970). The fastest reactions were made to the last (fourth) item in the set, while RTs to the remaining items were longer and nearly equal.

When examined together, the two studies suggest that a set of integers has different properties when processed once immediately after presentation than it has when processed many times with longer delays between presentation and test. In the early stages of processing, the availability of items may be primarily influenced by the organization provided by the serial nature of the task (temporal organization). The results obtained by Morin et al (1967) demonstrate that the major determinant of retrieval time was serial

* This investigation was supported by $\mathrm{National}$ Science Foundation Grant GB-16729 awarded to Bowling Green State Uni crity. position-the most recent item was consistently recognized fastest. In a later stage of processing, retrieval may be much more dependent on preexperimental experiences and associations (relationships among set members). In these instances the typical serial position curve may not obtain. Instead, functions like those observed by DeRosa \& Morin (1970) may be found (interior items reacted to fastest). The two different forms of organization referred to above are quite similar to what Tulving (1968) describes as primary and secondary organization in free recall learning.

In the present study, positive sets of four digits were presented serially prior to a test or probe stimulus. Positive sets differed in organization, and the delay between presentation and probe varied from .5 up to $5.0 \mathrm{sec}$. With increasing delays the organization of the set may become increasingly important.

The Ss were 15 male undergraduates who each participated in two $2 \cdot \mathrm{h}$ experimental sessions. Stimuli were the digits 1.9 presented on the viewing face of an I.E.E. display cell mounted $3 \mathrm{ft}$ from $\mathrm{S}$. Verbal responses activated a voice-operated relay, which terminated a test item and keyed an electronic counter which displayed RTs to the nearest . $01 \mathrm{sec}$.

Each trial consisted of a serial presentation of a red flash (warning signal) followed by four to-be-remembered digits (positive set). Each stimulus was on $.3 \mathrm{sec}$ and off .2 sec. A variable retention interval of $.5,1.5,3.0$, or $5.0 \mathrm{sec}$ followed the last positive set digit. At the end of the retention interval, a test or probe digit was shown. The Ss were instructed to say "yes" if the probe was identical to any item in the positive set and "no" otherwise. A .5-sec warning signal was provided before each probe by the onset of an amber lamp centered above the display cell.

On the first experimental day, Ss were given 20 practice trials to familiarize them with the task. Over the two experimental sessions, each $S$ was given 1,248 trials divided among six lists. A list contained 52 items and was presented four times. Each of the six lists was shown twice in a session, and each of the four delay conditions was presented three times. Within a list the delay between the last positive item and the probe was constant.

Three basic types of positive sets were employed which varied in organization. Since they are similar to the types of sets used by Morin et al (1967), their terminology is used to describe the sets. A closed set is one in which the four digits of the positive set could be permuted so as to be consecutive. The difference between the largest and smallest members of a closed set was always three. The closed sets used were: $1,2,3,4 ; 3,4,5,6$; and $6,7,8,9$. An open set was one in which the digits could not be permuted into a consecutive set. The open-closed distinction is one in which organization is manipulated by changing the ordinal distance between set members. In an open set, this distance may be as great as eight (1 and 9 in positive set). Sets were further distinguished by the sequence in which the numerals of the positive set were presented. An increasing set was one in which each digit in the positive set was larger than the preceding digit. An irregular set was one in which the digits were presented in a random order, and consecutive runs were never greater than two. The increasing-irregular dimension refers to the organization provided by the order of presentation of positive set members. The three types of positive sets and examples of each follows: closed-increasing $(3,4,5,6)$, closed-irregular $(7,6,9,8)$, and open-irregular $(4,1,7,5)$.

Probe stimuli were identical to items in each serial position equally often, and each digit appeared equally often as a positive probe. The probability, over trials, that the probe was identical to an item in the positive set was .5 .

RESULTS AND DISCUSSION

Figure 1 presents RT data for positive responses to each of the three types of sets under each delay condition. Each curve is a mean of 15 individual curves. The individual data were based on mean RTs for correct responses. The error rate over the entire experiment was $6.9 \%$.

A striking feature of the data in Fig. 1 is the clear superiority, in terms of RT, of closed sets over open sets. Reactions to digits in closed sets averaged approximately 50 msec faster than digits drawn from open sets. This finding holds for all delay conditions. The analysis performed on the data in Fig. 1 indicated that type of set was a highly reliable variable, 


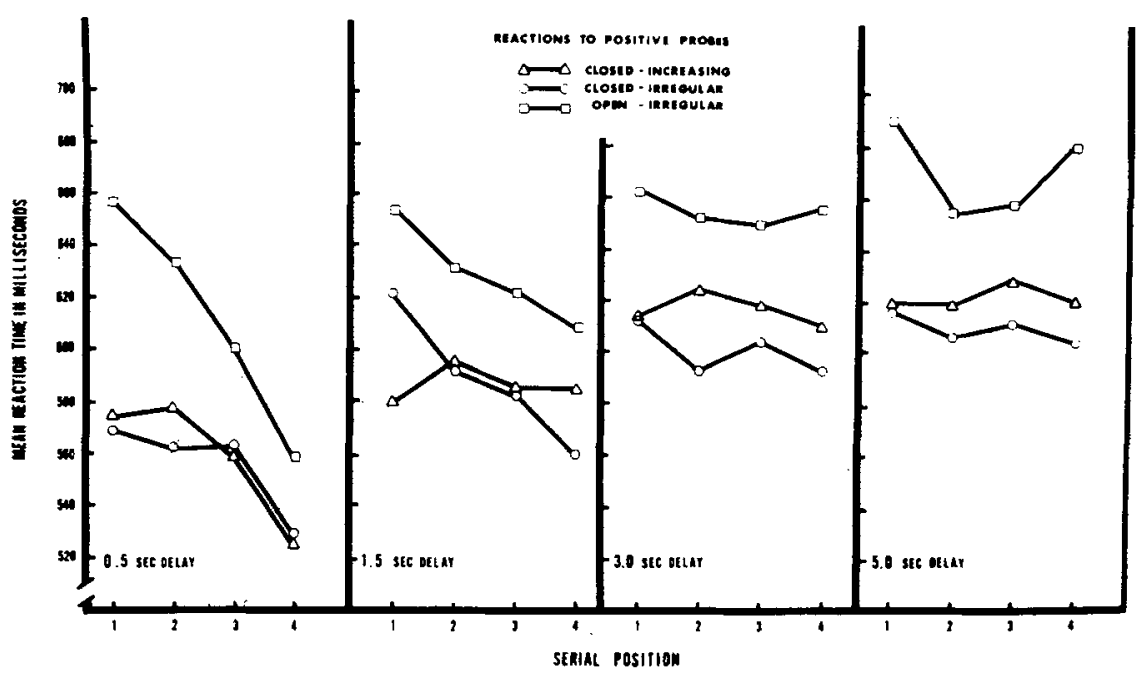

Fig. 1. Mean RT in milliseconds as a function of serial position in the positive set.

$F(2,28)=47.39, p<.01$. In addition, delay and serial position were both significant variables, $F(3,42)=53.99$, $\mathrm{p}<.01$, and $\mathrm{F}(3,42)=16.44, \mathrm{p}<.01$, respectively.

In an attempt to gain a better understanding of the influence of the delay and set organization on retrieval, separate analyses were conducted on the data at each delay interval. Particular attention was given to the influence of the delay on the serial position curves for the three different types of sets.

The presence of consistent differences in serial position curves for the three different types of positive set would be reflected in reliable Type of Set by Serial Position interactions. This interaction was indeed significant with delays of .5 and $1.5 \mathrm{sec}$ : $F(6.84)=7.721, \quad p<.01, \quad$ and $F(6,84)=2.906, p<.05$, respectively. These interactions reflect, in part, the greater influence of temporal factors on the serial position curves of unorganized sets. For example, at both the .5- and 1.5-sec delays, an open set produces a clear negative relationship between serial position and RT. Items closest to the probe are reacted to fastest. A similar but less dramatic recency effect is evidenced for the two closed sets at the .5-sec delay. Although the functions of both of these sets have a negative slope, the slope of the open set $(-33.00 \mathrm{msec}$ per position) is twice as great as the average of the two closed sets $(-15.33)$. At a delay of $1.5 \mathrm{sec}$, the open-irregular set and the closed-irregular set both still exhibit a negative slope: -15.67 and $-19.67 \mathrm{msec}$ per position, respectively. The closed-increasing set is nearly flat.
When the delay is increased to $3.0 \mathrm{sec}$, all functions are essentially flat. The interaction of Type of Set by Serial Position was not significant, $F(6,84)=1.009, p>.05$. With a delay of $5.0 \mathrm{sec}$, the functions for closed sets remain flat. There is no evidence of any effect of serial position with these two sets. ${ }^{1}$ The open set, on the other hand, has an unexpected U-shaped serial position curve, much like that observed by DeRosa \& Morin (1970) for their closed sets. Although the observed curves in the fourth panel of Fig. 1 are not parallel, the interaction of Type of Set by Serial Position was not significant, $F(6,84)=1.777$, $p>.05$. A more analytical analysis performed on the curve for an open-irregular set indicated that the quadratic component of the variance associated with serial position was significant, $F(1,14)=13.010, p<.01$. Reactions to items in the first and last position in the series are elevated above the level of reactions to items presented in the middle of the sequence. Attempts to rationalize this result all fail to provide a reasonable explanation for the result.

The findings for positive probes demonstrate a general tendency for temporal organization to be an important determinant of retrieval for only the first few seconds after presentation. Within $3 \mathrm{sec}$, all serial position curves are flat. The results do demonstrate that retrieval is influenced by set composition in at least two ways. First, the highly organized closed-increasing set is influenced by temporal organization for the shortest period of time. One and one-half seconds after the last positive set item is shown, the serial position curve for closed-increasing sets is flat. This is not true for the closed-irregular and the open-irregular sets. Both exhibit a recency effect. A second consequence of organization concerns the open-closed distinction. Closed sets possess some feature which results in faster RTs than those observed for open sets. Regardless of the shape of the serial position curves, RTs for closed sets are approximately $50 \mathrm{msec}$ faster than reactions to open sets. This finding holds for all delay intervals.

Although the primary purpose of this investigation was an examination of reactions to positive probes as a function of serial position, it is possible to evaluate reactions to negative probes. Previous studies (DeRosa \& Morin, 1970; Morin et al, 1967) have demonstrated that reactions to negative elements are influenced by an item's proximity to the positive set. Data for negative responses are shown in Fig. 2. Here RT is plotted as a function of a numeral's remoteness (the absolute difference between a numeral and the nearest member of the positive set) from the positive set. For example, with a positive set $(3,4,5,6)$ the digits in the negative set $(1,2,7,8,9)$ would be at a remoteness of $2,1,1,2$, and 3 , respectively. Morin et al (1967) and DeRosa \& Morin (1970) both found that RTs for negative responses were a decreasing function of remoteness - the greater the item's remoteness the faster S's reaction to that item. This finding is repeated in the present study.

In Fig. 2 data are plotted for correct reactions to negative items when positive sets were closed. Data for negative reactions when positive sets were open are not shown since a remoteness of 2 or more was infrequent. Data are shown for each delay condition. Reaction time did increase as a function of delay. The means for negative responses were $594,602,617$, and 617 msec for the $.5-, 1.5-, 3.0^{-}$, and 5.0 -sec delays, respectively. The analysis performed on the means indicated that delay was a reliable variable, $F(3,42)=7.28$, $\mathrm{p}<.01$. The interaction of delay with all other variables was not significant, indicating that the pattern of results is similar across each of the delay intervals. At all delays RT decreases as a function of remoteness. Remoteness is a highly significant variable, $\mathbf{F}(3,42)=34.24, \quad \mathbf{p}<.01$. Type of positive set was not significant, but its interaction with remoteness was significant, $F(4,56)=3.04, p<.05$. This interaction appears to be due primarily to the long RTs for items at a remoteness of 1 when positive sets were closed and increasing. In all four panels of Fig. 2, reactions to negative 


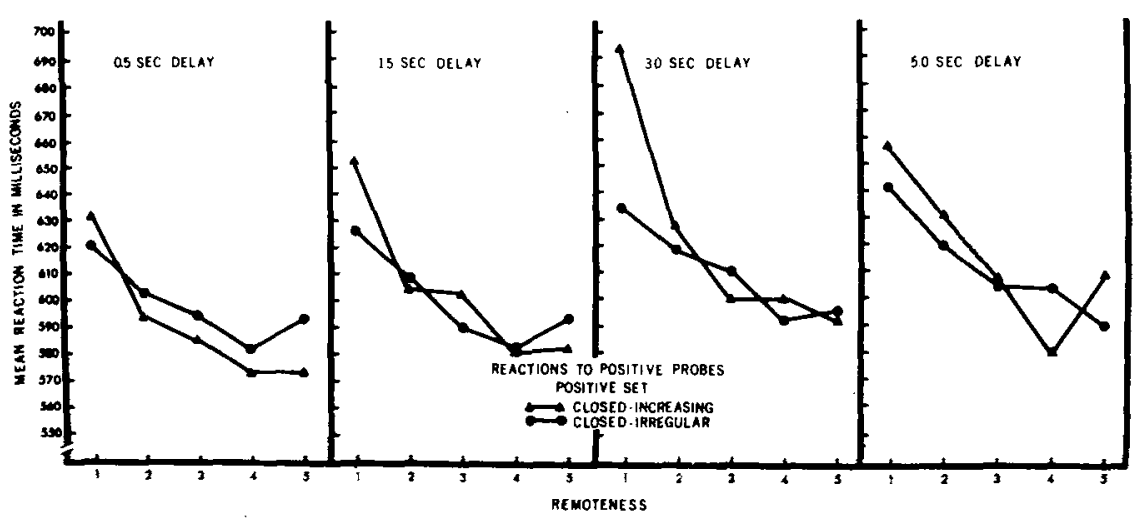
set.

Fig. 2. Mean RT in milliseconds as a function of remoteness from the positive probes at a remoteness of 1 are longest when the positive set is closed and increasing. This is not the case for elements at a remoteness greater than 1. Here the two curves tend to be much more comparable.

Although the results for negative reactions should be viewed with some caution since all digits did not appear equally often at each remoteness, the results are remarkably consistent with previous findings. In three markedly different experimental situations remoteness has been shown to be an important determinant of RTs to negative probes. In addition, variation due to remoteness is operative regardless of the pattern of results observed for positive responses. It appears that the finding is quite general and not affected by temporal organization to the degree which positive reactions are.

The remoteness effect illustrates the fact that the categorization process (yes-no) is influenced by features of stimuli which are not physical in nature. The features of negative elements which produce a retardation of reactions to items closest to the positive set are difficult to specify, but it is conceivable that one feature a digit may possess is its position within an ordered continuum (its magnitude). The positive set may define a region of a particular magnitude, and negative digits close in magnitude to the positive set are more similar to elements in the positive set than are elements which are more remote. This similarity might result in some form of response competition leading to slower reactions.

In summary, the results demonstrate that retrieval from a set of subspan items is influenced by temporal variables for a short time after presentation. In general, this influence is greatest and longer lasting when alternative means of organization are not available. After $3 \mathrm{sec}$ temporal organization is not an important determinant of retrieval time. Within the limits of the present investigation, no evidence was found to indicate that the U-shaped functions observed by DeRosa \& Morin (1970) were simply the result of a more permanent storage of that their result for positive probes may have been due to the fact that information. Instead, it seems likely
Students of form constancy have proposed at least two alternative methods by which the $S$ might determine the objective shape of a rotated object. Koffka's "invariance hypothesis" supposes tha judgments of the objective shape are determined by an analytic process of combining the retinal image shape and the perceived slant of the object. Thus, errors in judgments of the objective shape result from errors in perception of the slant or rotation. Despite attempts by Stravrianos (1945), Beck \& Gibson (1955), and Epstein, Bontrager, \& Park (1962) to demonstrate the covariance of these errors, there remain seemingly unsystematic variations in the S's judgments of objective shape which do not covary with judgments of slant. However, it may be that explicit judgments of shape and slant, taken separately, do

*This experiment was supported in whole by Washington State University Graduate School research funds. The auth or wishes to thank Constance $\mathbf{C}$. Kaess and Ronald Slater for assistance in data collection and reduction. sets did not change from trial to trial. Reactions to negative probes were influenced by remoteness and the result was independent of temporal variables.

\section{REFERENCES}

DeROSA, D. V., \& MORIN, R. E. Recognition reaction time for digits in consecutive and nonconsecutive memorized sets. Journal of Experimental Psychology, 1970, 83, 472-479.

MORIN, R. E., DeROSA, D. V., \& STULTZ, $V$. Recognition memory and reaction time. Acta Psychologica, 1967, 27, 298-305.

TULVING, E. Theoretical issues in free recall. In $T, R$. Dixon and D. L. Horton (Eds.) Verbal behavior and general behavior theory. Englewood Cliffs, N.J: Prentice-Hall, 1968. Pp. 2-36. NOTE

1. In a closed-increasing set, a digit's serial position in the series is confounded with its ordinal value in the set. This is not the case for closed-irregular or open-irregular sets. The RTs for items in these sets were rearranged, using ordinal value as an independent variable. For example, with a positive set $(6,3,5,4)$ the ordinal value of items in the set would be $4,1,3$, and 2 , respectively. The resulting mean RTs were nearly equal.

\title{
Analytic and intuitive perceivers in the form constancy task
}

\author{
DALE W. KAESS* \\ Washington State University, Pullman, Wash. 99163
}

Two alternatives for the method used by Ss to solve the form constancy task were tested by varying the range of angles to which rectangles were rotated. Results indicate that response consistency improves as the angles become less discriminable, contrary to Koffka's invariance hypothesis. Thus, an alternative intuitive mode of perceiver operation is supported.

not correspond to that which the $S$ actually perceives in the form constancy experiment.

Hake, Faust, McIntyre, \& Murray (1967) suggest an alternative mode of perceiver operation in the constancy situation. They suggest the "nondiscriminating," or intuitive perceptual mode, by which the $S$ does not determine separately the retinal image shape and the apparent slant. Rather, the perceiver simply obtains an intuitive shape-at-a-slant judgment.

A major difference between the two models of perceiver operation is the role of the angles to which the forms are rotated. When forms are presented at several discriminably different angles of rotation, the analytic perceiver should not perform well, due to the increased confusion in his judgment of slant. The nonanalytical or intuitive perceiver's performance should show the opposite trend with decreases of angle variation, however. To the intuitive perceiver, angle variation is basically "error variance," which must be overcome to achieve veridical perception of objective 